Sādhanā Vol. 37, Part 4, August 2012, pp. 461-480. (C) Indian Academy of Sciences

\title{
Buckling analysis and small scale effect of biaxially compressed graphene sheets using non-local elasticity theory
}

\author{
S C PRADHAN
}

Department of Aerospace Engineering, Indian Institute of Technology, Kharagpur 721 302, India

e-mail: scp.aero@gmail.com

MS received 11 October 2010; revised 30 March 2012; accepted 13 April 2012

\begin{abstract}
In this paper, buckling analysis of biaxially compressed graphene sheets with non-local elasticity theory is reported. The equations of motion for graphene sheet are derived using non-local local elasticity theory. Levy's approach has been used to solve the governing equations for various boundary conditions of the graphene sheet. Present results from Levy's solution agree with the results for all edges simply supported available in the literature. Further, the effect of the (i) non-local parameter, (ii) size of the graphene sheet and (iii) various boundary conditions on the critical buckling loads of the graphene sheets are investigated. It is observed that non-local parameter and boundary conditions significantly influence the critical buckling loads of the small size graphene sheets.
\end{abstract}

Keywords. Buckling; graphene sheet; non-local elasticity theory; Levy’s solution.

\section{Introduction}

Nanomaterials are known to have remarkable properties and hence have attracted much research interest. Since the discovery of fullerenes (Kroto 1985) and carbon nanotubes (Iijima 1991), nanomaterials have attracted a great deal of attention of the scientific community due to their outstanding mechanical, chemical and electronic properties (Gibson et al 2007). Applications of these nanostructures such as in MEMS/NEMS are dependent on the thorough understanding of their mechanical properties. Also, buckling of nanoplates (graphene sheets) is very important to predict the strength and failure of graphene sheets-based composites. Controlling experiments in nanoscale is difficult, hence theoretical analysis of nanostructures becomes important concerning application of nanostructures. Due to the discreteness of structures of nanomaterials, atomistic methods (Ball 2001; Baughman et al 2002) such as molecular dynamics, density functional theory, etc. are attempted to study the structural behaviour of the nanostructures. The above atomic methods, due to computational complexity, are limited to systems with a small number of molecules and atoms and therefore are restricted to the study of small-scale modelling. Hence, continuum modelling of nanostructures has received a great attention. The recently discovered graphene (Novoselov et al 2004), the two-dimensional carbon nanostructure has sparked a 
growing interest among scientists. It is believed that the analysis of graphene sheets is a fundamental issue in the study of nanomaterials because many of the carbon-based nanostructures. For example, carbon nanotubes (Iijima 1991), fullerenes (Kroto 1985), nanorings (Kong et al 2004), etc. are viewed as deformed graphene sheet. Further, some remarkable mechanical properties of graphene sheets reveal that they have potential for creating novel ultra strength composite materials (Thostenson et al 2001). Besides this, (Stankovich et al 2006) have recently achieved a valuable method of preparing graphene sheets for mass production usages. The reduced cost implies a future possibility of employing graphene sheets for general applications.

Despite above factors, there has been little research in the modelling of graphene sheets by the continuum method, compared to the studies on nanotubes (Ghorbanpour Arani et al 2008; Wang et al 2008; Phadikar \& Pradhan 2008; Wang \& Vardan 2006; Pradhan \& Murmu 2009c; Hsu 2010; Karaoglu \& Aydogdu 2010; Pradhan 2012). Research on graphene sheets has been mainly concerned with vibration of graphene sheets, and a few on buckling (Pradhan 2009; Pradhan \& Murmu 2009c, d, 2010a, b; Pradhan \& Sahu 2010; Pradhan \& Murmu 2009a; Pradhan \& Phadikar 2009a). Behfar \& Naghdabadi (2005) considered graphene sheet as an orthotropic, thin continuum plate and computed its natural frequencies. Further, most analyses employ the local elasticity theory. Due to the structural discreteness of the graphene sheet, these methods would not be able to predict the true structural response of nanostructures. This is because in this small scale length, the material microstructure can be important and this discrete structure can no longer be homogenized into a continuum. Hence, the non-local effect becomes important in capturing this structural discreteness. Further, there is little research on the effect of various boundary conditions on buckling of graphene sheets.

The basic difference between classical elasticity theory and non-local elasticity theory of Eringen (2002) is based on the definition of stress. In local elasticity, stress at a point is a function of strain at that point only, whereas in non-local elasticity, the stress at any point is a function of strains at all points in the continuum. Hence, the non-local theory contains information about long range forces about atoms and thus internal scale length is introduced in the formulation. Though non-local elasticity has been applied to various fields of physics including lattice dispersion of elastic waves, dislocation mechanics (Eringen 1983), etc. Peddieson et al (2003) suggested analysis of nanostructures based on non-local elasticity theory. Since then many researchers ( $\mathrm{Lu}$ et al 2007) have applied non-local continuum theories to model carbon nanotubes or similar nanobeam like structures. Duan \& Wang (2007) reported bending analysis of circular graphene sheets based on non-local elasticity theory. Some research works have been reported on the thermal effect on buckling of nanotubes (Ghorbanpour Arani et al 2011), vibration of nanotubes (Ghorbanpour Arani et al 2010), multilayered graphene sheets (Kitipornchai et al 2005; Liew et al 2006; Pradhan \& Phadikar 2009a, b, 2010), nonhomogenous nanotubes (Phadikar \& Pradhan 2009), non-local beams on Winkler foundations (Pradhan \& Murmu 2009b; Murmu \& Pradhan 2008) and non-local tapered beams (Pradhan \& Sarkar 2009).

In the present work, a continuum model based on non-local elasticity theory has been employed for computing critical buckling loads of graphene sheets. Material is considered as an isotropic nanoplate, and the model is utilized to study the small scale effect on graphene sheets for various boundary conditions.

\section{Formulation}

\subsection{Single layered graphene sheet}

Though graphene sheets are generally found in multi-layered form, Stankovich et al (2006) reported successful extraction of single layered non-local graphene sheets from graphite. 


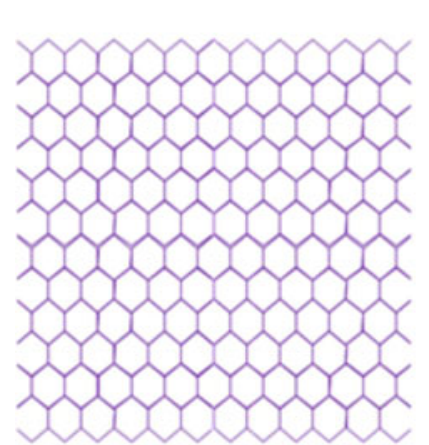

(a)

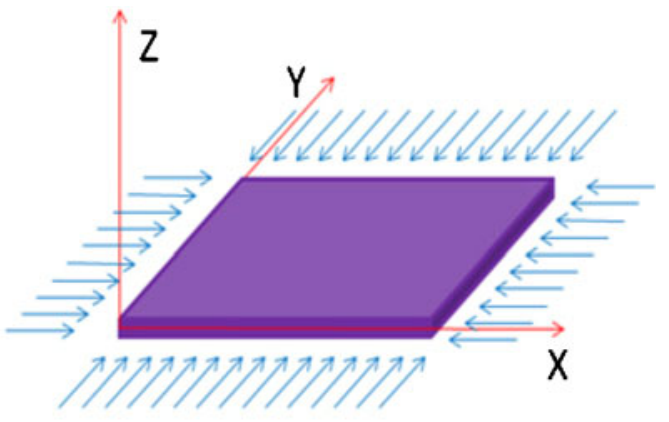

(b)

Figure 1. Single layered graphene sheet: (a) discrete model, (b) continuum model.

Figure 1a shows a discrete rectangular graphene sheet and figure $1 \mathrm{~b}$ shows its equivalent continuum model. Kitipornchai et al (2005) considered graphene sheet as isotropic material in their continuum model. In the present work, the graphene sheet model has been assumed to be isotropic. The coordinate system used for the graphene sheet is shown in figure 1b. Origin is chosen at one corner of the graphene sheet. The $x, y$ and $z$ coordinates of the axes are taken along the length, width and thickness of the graphene sheet. Following stress resultants are used in the present formulation

$$
\begin{aligned}
N_{0}^{x x} & =\int_{-h / 2}^{h / 2} \sigma_{x x} d z, \quad N_{0}^{y y}=\int_{-h / 2}^{h / 2} \sigma_{y y} d z, \quad N_{0}^{x y}=\int_{-h / 2}^{h / 2} \sigma_{x y} d z \\
M_{1}^{x x} & =\int_{-h / 2}^{h / 2} z \sigma_{x x} d z, \quad M_{1}^{y y}=\int_{-h / 2}^{h / 2} z \sigma_{y y} d z, \quad M_{1}^{x y}=\int_{-h / 2}^{h / 2} z \sigma_{x y} d z .
\end{aligned}
$$

The displacement field is written as

$$
u_{x}=u(x, y, t)-z \frac{\partial w}{\partial x}, u_{y}=v(x, y, t)-z \frac{\partial w}{\partial y} \text { and } u_{z}=w(x, y, t) .
$$

Here $u, v$ and $w$ denote displacement along $x, y$ and $z$ directions, respectively.

The strains are expressed as

$$
\begin{aligned}
& \varepsilon_{x x}=\frac{\partial u}{\partial x}-z \frac{\partial^{2} w^{c}}{\partial x^{2}}, \quad \varepsilon_{y y}=\frac{\partial v}{\partial x}-z \frac{\partial^{2} w^{c}}{\partial y^{2}}, \quad \varepsilon_{z z}=0, \\
& \varepsilon_{x y}=\frac{1}{2}\left(\frac{\partial u}{\partial y}+\frac{\partial v}{\partial x}-2 z \frac{\partial^{2} w^{c}}{\partial x y}\right), \varepsilon_{x z}=0, \quad \varepsilon_{y z}=0 .
\end{aligned}
$$

According to Eringen (2002) the non-local constitutive behaviour of a Hookean solid can be represented by the following differential constitutive relation

$$
\left(1-\mu \nabla^{2}\right) \sigma=t
$$


Here $\mu$ is the non-local parameter; $\boldsymbol{t}$ is the macroscopic stress tensor at a point which is related to strain by generalized Hooke's law

$$
t(x)=S(x): \varepsilon(x),
$$

where $S$ is the fourth order elasticity tensor and ':' denotes the double dot product.

It can be noted that non-local behaviour enters through the constitutive relations. Principle of virtual work is independent of constitutive relations. So this can be applied to derive the equilibrium equations of the non-local plates.

Using the principle of virtual work, following equilibrium equations can be obtained (Reddy 1997)

$$
\begin{gathered}
\frac{\partial N_{0}^{x x}}{\partial x}+\frac{\partial N_{0}^{x y}}{\partial y}=m_{0} \frac{\partial^{2} u}{\partial t^{2}}, \\
\frac{\partial N_{0}^{y y}}{\partial y}+\frac{\partial N_{0}^{x y}}{\partial x}=m_{0} \frac{\partial^{2} v}{\partial t^{2}}, \\
\frac{\partial^{2} M_{1}^{x x}}{\partial x^{2}}+2 \frac{\partial^{2} M_{1}^{x y}}{\partial x \partial y}+\frac{\partial^{2} M_{1}^{y y}}{\partial y^{2}}+q+\frac{\partial}{\partial x}\left(N_{0}^{x x} \frac{\partial w^{c}}{\partial x}\right)+\frac{\partial}{\partial y}\left(N_{0}^{y y} \frac{\partial w^{c}}{\partial y}\right) \\
+\frac{\partial}{\partial x}\left(N_{0}^{x y} \frac{\partial w^{c}}{\partial y}\right)+\frac{\partial}{\partial y}\left(N_{0}^{x y} \frac{\partial w^{c}}{\partial x}\right)=0 .
\end{gathered}
$$

Here, $h$ denotes the height of the plate; $\sigma_{x x}, \sigma_{y y}, \sigma_{z z}, \sigma_{x y}, \sigma_{y z}$ and $\sigma_{x z}$ denote the stress tensors. Using Eq. (4), the plane stress constitutive relation of a non-local plate becomes

$$
\left\{\begin{array}{l}
\sigma_{x x} \\
\sigma_{y y} \\
\sigma_{x y}
\end{array}\right\}-\mu \nabla^{2}\left\{\begin{array}{c}
\sigma_{x x} \\
\sigma_{y y} \\
\sigma_{x y}
\end{array}\right\}=\left[\begin{array}{ccc}
E /\left(1-v^{2}\right) & v E /\left(1-v^{2}\right) & 0 \\
v E /\left(1-v^{2}\right) & E /\left(1-v^{2}\right) & 0 \\
0 & 0 & 2 G
\end{array}\right]\left\{\begin{array}{l}
\varepsilon_{x x} \\
\varepsilon_{y y} \\
\varepsilon_{x y}
\end{array}\right\}
$$

Here, $E, G$ and $v$ are Young's modulus, shear modulus and Poisson's ratio, respectively. Using strain displacement relationship of Eq. (3), stress-strain relationship of Eq. (7) and stress resultants definition of Eq. (1), we can express stress resultants in terms of displacements as follows

$$
\begin{gathered}
M_{1}^{x x}-\mu \nabla^{2} M_{1}^{x x}=-D\left(\frac{\partial^{2} w}{\partial x^{2}}+v \frac{\partial^{2} w}{\partial y^{2}}\right), \\
M_{1}^{y y}-\mu \nabla^{2} M_{1}^{y y}=-D\left(\frac{\partial^{2} w}{\partial x^{2}}+\frac{\partial^{2} w}{\partial y^{2}}\right), \\
M_{1}^{x y}-\mu \nabla^{2} M_{1}^{x y}=-D(1-v) \frac{\partial^{2} w}{\partial x \partial y} .
\end{gathered}
$$


$\mathrm{D}$ is the bending rigidity and is defined as

$$
D=\frac{E h^{3}}{12\left(1-v^{2}\right)} .
$$

Using Eq. (6) and Eq. (8) following governing equations are obtained in terms of displacements

$$
\begin{aligned}
- & D \frac{\partial^{4} w}{\partial x^{4}}-2 D \frac{\partial^{4} w}{\partial x^{2} \partial y^{2}}-D \frac{\partial^{4} w}{\partial y^{4}} \\
+ & \mu \nabla^{2}\left[-q-\frac{\partial}{\partial x}\left(N_{0}^{x x} \frac{\partial w}{\partial x}\right)-\frac{\partial}{\partial y}\left(N_{0}^{y y} \frac{\partial w}{\partial y}\right)-\frac{\partial}{\partial x}\left(N_{0}^{x y} \frac{\partial w}{\partial y}\right)-\frac{\partial}{\partial y}\left(N_{0}^{x y} \frac{\partial w}{\partial x}\right)\right] \\
+ & q+\frac{\partial}{\partial x}\left(N_{0}^{x x} \frac{\partial w}{\partial x}\right)+\frac{\partial}{\partial y}\left(N_{0}^{y y} \frac{\partial w}{\partial y}\right)+\frac{\partial}{\partial x}\left(N_{0}^{x y} \frac{\partial w}{\partial y}\right) \\
+ & \frac{\partial}{\partial y}\left(N_{0}^{x y} \frac{\partial w}{\partial x}\right)=0 .
\end{aligned}
$$

It can be seen that the corresponding local elasticity equation written in (Reddy 1997) is recovered by putting $\mu=0$ in the above equations.

\subsection{Levy's solution}

An isotropic and homogeneous rectangular graphene sheet with uniform thickness $h$, length $a$, width $b$, Young's modulus $E$, Poisson's ratio $v$, and the rectangular cartesian coordinate system as shown in figure $1 \mathrm{~b}$ is considered. In this work, the graphene sheets which are simply supported on two opposite edges with any combination of simply supported, clamped or free boundary conditions at the remaining two edges of the graphene sheet are considered. The following expansions of various generalized displacements have been assumed in (Timoshenko \& Gere 1985)

$$
w=f(y) \sin \frac{m \pi x}{a} .
$$

The above is justified as it satisfies the simply supported boundary conditions at $x=0$ and $x=a$.

With this expansion, the differential eq. (10) is written as

$$
\begin{gathered}
-D \frac{m^{4} \pi^{4}}{a^{4}} \sin \frac{m \pi x}{a} f(y)+2 D \frac{d^{2} f(y)}{d y^{2}} \frac{m^{2} \pi^{2}}{a^{2}} \sin \frac{m \pi x}{a}-D \frac{d^{4} f(y)}{d y^{4}} \sin \frac{m \pi x}{a} \\
+\mu \nabla^{2}\left[-q-\frac{\partial}{\partial x}\left(N_{0}^{x x} f(y) \frac{m \pi}{a} \cos \frac{m \pi x}{a}\right)-\frac{\partial}{\partial y}\left(N_{0}^{y y} \frac{d f(y)}{d y} \sin \frac{m \pi x}{a}\right)\right. \\
\left.\quad-\frac{\partial}{\partial x}\left(N_{0}^{x y} \frac{d f(y)}{d y} \sin \frac{m \pi x}{a}\right)-\frac{\partial}{\partial y}\left(N_{0}^{x y} f(y) \frac{m \pi}{a} \cos \frac{m \pi x}{a}\right)\right] \\
+q+\frac{\partial}{\partial x}\left(N_{0}^{x x} f(y) \frac{m \pi}{a} \cos \frac{m \pi x}{a}\right)+\frac{\partial}{\partial y}\left(N_{0}^{y y} \frac{d f(y)}{d y} \sin \frac{m \pi x}{a}\right) \\
+\frac{\partial}{\partial x}\left(N_{0}^{x y} \frac{d f(y)}{d y} \sin \frac{m \pi x}{a}\right)+\frac{\partial}{\partial y}\left(N_{0}^{x y} f(y) \frac{m \pi}{a} \cos \frac{m \pi x}{a}\right)=0 .
\end{gathered}
$$


It is assumed that the plate is free from any transverse loadings. So, $N_{0}^{x y}=q=0$. Eq. (11) is reduced to

$$
\begin{aligned}
- & D \frac{m^{4} \pi^{4}}{a^{4}} \sin \frac{m \pi x}{a} f(y)+2 D \frac{d^{2} f(y)}{d y^{2}} \frac{m^{2} \pi^{2}}{a^{2}} \sin \frac{m \pi x}{a}-D \frac{d^{4} f(y)}{d y^{4}} \sin \frac{m \pi x}{a} \\
+ & \mu \nabla^{2}\left[-\frac{\partial}{\partial x}\left(N_{0}^{x x} f(y) \frac{m \pi}{a} \cos \frac{m \pi x}{a}\right)-\frac{\partial}{\partial y}\left(N_{0}^{y y} \frac{d f(y)}{d y} \sin \frac{m \pi x}{a}\right)\right] \\
& +\frac{\partial}{\partial x}\left(N_{0}^{x x} f(y) \frac{m \pi}{a} \cos \frac{m \pi x}{a}\right)+\frac{\partial}{\partial y}\left(N_{0}^{y y} \frac{d f(y)}{d y} \sin \frac{m \pi x}{a}\right)=0 .
\end{aligned}
$$

Simplifying Eq. (12)

$$
\begin{gathered}
-D \frac{m^{4} \pi^{4}}{a^{4}} \sin \frac{m \pi x}{a} f(y)+2 D \frac{d^{2} f(y)}{d y^{2}} \frac{m^{2} \pi^{2}}{a^{2}} \sin \frac{m \pi x}{a}-D \frac{d^{4} f(y)}{d y^{4}} \sin \frac{m \pi x}{a} \\
+\mu\left[\left(-N_{0}^{x x} f(y) \frac{m^{4} \pi^{4}}{a^{4}} \sin \frac{m \pi x}{a}\right)+\left(N_{0}^{y y} \frac{d^{2} f(y)}{d y^{2}} \frac{m^{2} \pi^{2}}{a^{2}} \sin \frac{m \pi x}{a}\right)\right. \\
\left.\quad+\left(N_{0}^{x x} \frac{d^{2} f(y)}{d y^{2}} \frac{m^{2} \pi^{2}}{a^{2}} \sin \frac{m \pi x}{a}\right)-\left(N_{0}^{y y} \frac{d^{4} f(y)}{d y^{4}} \sin \frac{m \pi x}{a}\right)\right] \\
-\left[\left(N_{0}^{x x} f(y) \frac{m^{2} \pi^{2}}{a^{2}} \sin \frac{m \pi x}{a}\right)-\left(N_{0}^{y y} \frac{d^{2} f(y)}{d y^{2}} \sin \frac{m \pi x}{a}\right)\right]=0 .
\end{gathered}
$$

Since $\sin \frac{m \pi x}{a}$ is a common factor,

$$
\begin{aligned}
& -D \frac{m^{4} \pi^{4}}{a^{4}} f(y)+2 D \frac{d^{2} f(y)}{d y^{2}} \frac{m^{2} \pi^{2}}{a^{2}}-D \frac{d^{4} f(y)}{d y^{4}} \\
& +\mu\left[\left(-N_{0}^{x x} f(y) \frac{m^{4} \pi^{4}}{a^{4}}\right)+\left(N_{0}^{y y} \frac{d^{2} f(y)}{d y^{2}} \frac{m^{2} \pi^{2}}{a^{2}}\right)+\left(N_{0}^{x x} \frac{d^{2} f(y)}{d y^{2}} \frac{m^{2} \pi^{2}}{a^{2}}\right)-\left(N_{0}^{y y} \frac{d^{4} f(y)}{d y^{4}}\right)\right] \\
& -\left[\left(N_{0}^{x x} f(y) \frac{m^{2} \pi^{2}}{a^{2}}\right)-\left(N_{0}^{y y} \frac{d^{2} f(y)}{d y^{2}}\right)\right]=0 .
\end{aligned}
$$

On simplification

$$
\begin{aligned}
& \frac{d^{4} f(y)}{d y^{4}}-\frac{d^{2} f(y)}{d y^{2}} \frac{1}{\left[D+\mu N_{0}^{y y}\right]}\left[2 D \frac{m^{2} \pi^{2}}{a^{2}}+\mu\left(N_{0}^{y y} \frac{m^{2} \pi^{2}}{a^{2}}+N_{0}^{x x} \frac{m^{2} \pi^{2}}{a^{2}}\right)+N_{0}^{y y}\right] \\
& +f(y) \frac{1}{\left[D+\mu N_{0}^{y y}\right]}\left[D \frac{m^{4} \pi^{4}}{a^{4}}+\mu\left(N_{0}^{x x} \frac{m^{4} \pi^{4}}{a^{4}}\right)+\left(N_{0}^{x x} \frac{m^{2} \pi^{2}}{a^{2}}\right)\right]=0 .
\end{aligned}
$$

Eq. (15) is an ordinary differential equation of the form

$$
\frac{d^{4} f(y)}{d y^{4}}-\frac{d^{2} f(y)}{d y^{2}} \lambda+f(y) \zeta=0
$$


where

$$
\begin{aligned}
& \lambda=\frac{1}{\left[D+\mu N_{0}^{y y}\right]}\left[2 D \frac{m^{2} \pi^{2}}{a^{2}}+\mu\left(N_{0}^{y y} \frac{m^{2} \pi^{2}}{a^{2}}+N_{0}^{x x} \frac{m^{2} \pi^{2}}{a^{2}}\right)+N_{0}^{y y}\right] \\
& \zeta=\frac{1}{\left[D+\mu N_{0}^{y y}\right]}\left[D \frac{m^{4} \pi^{4}}{a^{4}}+\mu\left(N_{0}^{x x} \frac{m^{4} \pi^{4}}{a^{4}}\right)+\left(N_{0}^{x x} \frac{m^{2} \pi^{2}}{a^{2}}\right)\right] .
\end{aligned}
$$

Substituting $f(y)=e^{\frac{\psi y}{b}}$,

$$
\left(\frac{\psi}{b}\right)^{4} f(y)-\lambda\left(\frac{\psi}{b}\right)^{2} f(y)+\zeta f(y)=0 .
$$

As $f(y) \neq 0 \forall y$

$$
\left(\frac{\psi}{b}\right)^{4}-\lambda\left(\frac{\psi}{b}\right)^{2}+\zeta=0 .
$$

Solving this, four values of $\psi_{1,2,3,4}$ are obtained,

$$
\begin{aligned}
& \psi_{1,3}= \pm \sqrt{\left(\frac{b^{2} \lambda+\sqrt{b^{4} \lambda^{2}-4 \zeta b^{4}}}{2}\right)} \\
& \psi_{2,4}= \pm \sqrt{\left(\frac{b^{2} \lambda-\sqrt{b^{4} \lambda^{2}-4 \zeta b^{4}}}{2}\right)} .
\end{aligned}
$$

Hence $f(y)$ is expanded as

$$
\begin{aligned}
& A \cosh \left(\frac{\psi_{1} y}{b}\right)+B \sinh \left(\frac{\psi_{1} y}{b}\right)+C \cos \left(\frac{\psi_{2} y}{b}\right)+D \sin \left(\frac{\psi_{2} y}{b}\right) \\
& \text { and } w=\left[A \cosh \left(\frac{\psi_{1} y}{b}\right)+B \sinh \left(\frac{\psi_{1} y}{b}\right)+C \cos \left(\frac{\psi_{2} y}{b}\right)+D \sin \left(\frac{\psi_{2} y}{b}\right)\right]\left[\sin \frac{m \pi x}{a}\right] .
\end{aligned}
$$

Now applying a combination of any of the three boundary conditions (clamped, simply supported and free) at $y=0$ and $y=b$, four equations are obtained.

Uniform compressive edge loading of magnitude $P$ along $x$ axis and $k P$ along $y$ axis have been assumed as shown in figure 1. Hence,

$$
N_{0}^{x x}=-P, N_{0}^{y y}=-k P
$$

where $k$ is the loading factor.

For the simply supported boundary condition case

$$
\begin{aligned}
& w=0 \text { and } \frac{d^{2} w}{d y^{2}}+v \frac{d^{2} w}{d x^{2}}=0 \\
& A \cosh \left(\frac{\psi_{1} y}{b}\right)+B \sinh \left(\frac{\psi_{1} y}{b}\right)+C \cos \left(\frac{\psi_{2} y}{b}\right)+D \sin \left(\frac{\psi_{2} y}{b}\right)=0 .
\end{aligned}
$$




$$
\begin{aligned}
& A\left(\left(\frac{\psi_{1}}{b}\right)^{2}-\frac{m^{2} \pi^{2}}{a^{2}} v\right) \cosh \left(\frac{\psi_{1} y}{b}\right)+B\left(\left(\frac{\psi_{1}}{b}\right)^{2}-\frac{m^{2} \pi^{2}}{a^{2}} v\right) \sinh \left(\frac{\psi_{1} y}{b}\right) \\
& -C\left(\left(\frac{\psi_{2}}{b}\right)^{2}+\frac{m^{2} \pi^{2}}{a^{2}} v\right) \cos \left(\frac{\psi_{2} y}{b}\right)-D\left(\left(\frac{\psi_{2}}{b}\right)^{2}+\frac{m^{2} \pi^{2}}{a^{2}} v\right) \sin \left(\frac{\psi_{2} y}{b}\right)=0 .
\end{aligned}
$$

For the clamped boundary condition case

$$
\begin{aligned}
& w=0 \text { and } \frac{d w}{d y}=0 \\
& A \cosh \left(\frac{\psi_{1} y}{b}\right)+B \sinh \left(\frac{\psi_{1} y}{b}\right)+C \cos \left(\frac{\psi_{2} y}{b}\right)+D \sin \left(\frac{\psi_{2} y}{b}\right)=0 \\
& A\left(\frac{\psi_{1}}{b}\right) \cosh \left(\frac{\psi_{1} y}{b}\right)+B\left(\frac{\psi_{1}}{b}\right) \sinh \left(\frac{\psi_{1} y}{b}\right)-C\left(\frac{\psi_{2}}{b}\right) \sin \left(\frac{\psi_{2} y}{b}\right) \\
& \quad+D\left(\frac{\psi_{2}}{b}\right) \cos \left(\frac{\psi_{2} y}{b}\right)=0
\end{aligned}
$$

For the free boundary condition case

$$
\begin{aligned}
& \frac{d^{2} w}{d y^{2}}+v \frac{d^{2} w}{d x^{2}}=0 \text { and } \frac{d^{3} w}{d y^{3}}+(2-v) \frac{d^{3} w}{d x^{2} d y}=0 \\
& A\left(\left(\frac{\psi_{1}}{b}\right)^{2}-\frac{m^{2} \pi^{2}}{a^{2}} v\right) \cosh \left(\frac{\psi_{1} y}{b}\right)+B\left(\left(\frac{\psi_{1}}{b}\right)^{2}-\frac{m^{2} \pi^{2}}{a^{2}} v\right) \sinh \left(\frac{\psi_{1} y}{b}\right) \\
& \quad-C\left(\left(\frac{\psi_{2}}{b}\right)^{2}+\frac{m^{2} \pi^{2}}{a^{2}} v\right) \cos \left(\frac{\psi_{2} y}{b}\right)-D\left(\left(\frac{\psi_{2}}{b}\right)^{2}+\frac{m^{2} \pi^{2}}{a^{2}} v\right) \sin \left(\frac{\psi_{2} y}{b}\right)=0 . \quad \text { (25) } \\
& A\left(\left(\frac{\psi_{1}}{b}\right)^{3}-\frac{m^{2} \pi^{2}}{a^{2}}(2-v)\left(\frac{\psi_{1}}{b}\right)\right) \sinh \left(\frac{\psi_{1} y}{b}\right)+B\left(\left(\frac{\psi_{1}}{b}\right)^{3}-\frac{m^{2} \pi^{2}}{a^{2}}(2-v)\left(\frac{\psi_{1}}{b}\right)\right) \cosh \left(\frac{\psi_{1} y}{b}\right) \\
& +C\left(\left(\frac{\psi_{2}}{b}\right)^{3}+\frac{m^{2} \pi^{2}}{a^{2}}(2-v)\left(\frac{\psi_{2}}{b}\right)\right) \sin \left(\frac{\psi_{2} y}{b}\right) \\
& -D\left(\left(\frac{\psi_{2}}{b}\right)^{3}+\frac{m^{2} \pi^{2}}{a^{2}}(2-v)\left(\frac{\psi_{2}}{b}\right)\right) \cos \left(\frac{\psi_{2} y}{b}\right)=0 .
\end{aligned}
$$

Abbreviating,

$$
\begin{aligned}
& t_{1}=\frac{\psi_{1}}{b}, t_{2}=\frac{\psi_{2}}{b}, z=\frac{m^{2} \pi^{2}}{a^{2}} \\
& q_{1}=\cosh \left(\psi_{1}\right), q_{2}=\sinh \left(\psi_{1}\right), q_{3}=\cos \left(\psi_{2}\right), q_{4}=\sin \left(\psi_{2}\right) .
\end{aligned}
$$

Following six boundary conditions of the graphene sheet are considered in the present analysis.

(i) SSSS: Simply supported on all four sides, (ii) SSSC: Simply supported on three sides, $x=0, x=a, y=0$ and clamped on one side, $y=a$, (iii) SSSF: Simply supported on three 
sides, $x=0, x=a, y=0$ and Free on one side, $y=a$, (iv) SCSC: Simply supported on $x=0$ and $x=a$ and clamped on $y=0$ and $y=a$, (v) SCSF: Simply supported on $x=0$ and $x=a$, clamped on $y=0$ and free on $y=a$, (vi) SFSF: Simply supported on $x=0$ and $x=a$ and free on $y=0$ and $y=a$.

\subsection{SSSS Boundary condition}

As the graphene sheet is simply supported on all four sides, the simply supported boundary condition is applied at $y=0$ and $y=b$. The simply supported boundary conditions at $x=0$ and $x=a$ are already satisfied, as mentioned earlier by the choice of the displacement function. Hence, applying Eq. (19) and Eq. (20) at $y=0$ and $y=b$, four equations are obtained which are represented below in a matrix form

$$
\left[\begin{array}{l}
A \\
B \\
C \\
D
\end{array}\right]\left[\begin{array}{cccc}
1 & 0 & 1 & 0 \\
\left(t_{1}^{2}-z v\right) & 0 & -\left(t_{2}^{2}+z v\right) & 0 \\
q_{1} & q_{2} & q_{3} & q_{4} \\
\left(t_{1}^{2}-z v\right) q_{1} & \left(t_{1}^{2}-z v\right) q_{2} & -\left(t_{2}^{2}-z v\right) q_{3} & -\left(t_{2}^{2}-z v\right) q_{4}
\end{array}\right]=0 .
$$

For $w$ to have a non-trivial solution, i.e., in the case of buckling, A, B, C, D should not be zero. For this condition to be satisfied

$$
\left|\begin{array}{cccc}
1 & 0 & 1 & 0 \\
\left(t_{1}^{2}-z v\right) & 0 & -\left(t_{2}^{2}+z v\right) & 0 \\
q_{1} & q_{2} & q_{3} & q_{4} \\
\left(t_{1}^{2}-z v\right) q_{1} & \left(t_{1}^{2}-z v\right) q_{2} & -\left(t_{2}^{2}+z v\right) q_{3} & -\left(t_{2}^{2}+z v\right) q_{4}
\end{array}\right|=0
$$

\subsection{SSSC Boundary condition}

Similarly, applying the simply supported boundary condition at $y=0$ and clamped boundary condition at $y=b$, i.e., Eq. (19) and Eq. (20) at $y=0$, Eq. (21) and Eq. (22) at $y=b$, the following equation is obtained

$$
\left|\begin{array}{cccc}
1 & 0 & 1 & 0 \\
\left(t_{1}^{2}-z v\right) & 0 & -\left(t_{2}^{2}+z v\right) & 0 \\
q_{1} & q_{2} & q_{3} & q_{4} \\
t_{1} q_{2} & t_{1} q_{1} & -t_{2} q_{4} & t_{2} q_{3}
\end{array}\right|=0
$$

\subsection{SSSF Boundary condition}

In the same manner, simply supported boundary condition, i.e., Eq. (19) and Eq. (20) are applied at $y=0$ while the free boundary condition i.e., Eq. (23) and Eq. (24) are applied at $y=b$. The following equation is obtained

$$
\left|\begin{array}{cccc}
1 & 0 & 1 & 0 \\
\left(t_{1}^{2}-z v\right) & 0 & -\left(t_{2}^{2}+z v\right) & 0 \\
\left(t_{1}^{2}-z v\right) q_{1} & \left(t_{1}^{2}-z v\right) q_{2} & -\left(t_{2}^{2}+z v\right) q_{3} & -\left(t_{2}^{2}+z v\right) q_{4} \\
\left(t_{1}^{3}-z(2-v) t_{1}\right) q_{2} & \left(t_{1}^{3}-z(2-v) t_{1}\right) q_{1} & \left(t_{2}^{3}+z(2-v) t_{2}\right) q_{4} & -\left(t_{2}^{3}+z(2-v) t_{2}\right) q_{3}
\end{array}\right|=0 .
$$




\subsection{SCSC Boundary condition}

Again, clamped boundary condition, i.e., Eq. (19) and Eq. (20) are applied at $y=0$ and $y=b$ to obtain the following equation

$$
\left|\begin{array}{cccc}
1 & 0 & 1 & 0 \\
0 & t_{1} & 0 & t_{2} \\
q_{1} & q_{2} & q_{3} & q_{4} \\
t_{1} q_{2} & t_{1} q_{1} & -t_{2} q_{4} & t_{2} q_{3}
\end{array}\right|=0
$$

\subsection{SCSF Boundary condition}

Clamped boundary condition, i.e., Eq. (21) and Eq. (22) are applied at $y=0$ while the free boundary condition i.e., Eq. (23) and Eq. (24) are applied at $y=b$. The following equation is obtained

$$
\left|\begin{array}{cccc}
1 & 0 & 1 & 0 \\
0 & t_{1} & 0 & t_{2} \\
\left(t_{1}^{2}-z v\right) q_{1} & \left(t_{1}^{2}-z v\right) q_{2} & -\left(t_{1}^{2}+z v\right) q_{3} & -\left(t_{2}^{2}+z v\right) q_{4} \\
\left(t_{1}^{3}-z(2-v) t_{1}\right) q_{2} & \left(t_{1}^{3}-z(2-v) t_{1}\right) q_{1} & \left(t_{2}^{3}+z(2-v) t_{2}\right) q_{4} & -\left(t_{2}^{3}+z(2-v) t_{2}\right) q_{3}
\end{array}\right|=0 .
$$

\subsection{SFSF Boundary condition}

Free boundary condition, i.e., Eq. (23) and Eq. (24) are applied at $y=0$ and $y=b$ to obtain the following equation

$$
\left|\begin{array}{cccc}
\left(t_{1}^{2}-z v\right) & 0 & -\left(t_{1}^{2}+z v\right) & 0 \\
0 & \left(t_{1}^{3}-z(2-v) t_{1}\right) & 0 & -\left(t_{2}^{3}+z(2-v) t_{2}\right) \\
\left(t_{1}^{2}-z v\right) q_{1} & \left(t_{1}^{2}-z v\right) q_{2} & -\left(t_{1}^{2}+z v\right) q_{3} & -\left(t_{2}^{2}+z v\right) q_{4} \\
\left(t_{1}^{3}-z(2-v) t_{1}\right) q_{2} & \left(t_{1}^{3}-z(2-v) t_{1}\right) q_{1} & \left(t_{2}^{3}+z(2-v) t_{2}\right) q_{4} & -\left(t_{2}^{3}+z(2-v) t_{2}\right) q_{3}
\end{array}\right|=0 .
$$

Now, in Eqs (28-33) for the six boundary condition $\psi_{1}=f(P, a, m, \mu), \psi_{2}=g(P, a, m, \mu)$ and definition of $\psi_{1,2,3,4}$ are employed. Hence for each of the above six boundary conditions $P_{\text {critical }}$ are calculated numerically for various values of $a, m$, and $\mu$.

Table 1. Critical buckling load of equivalent simply supported nanobeam.

\begin{tabular}{llcc}
\hline $\mathrm{L} / \mathrm{h}$ & $\mu$ & $\bar{P}_{\text {critical }}$ (Reddy 2007) & $\bar{P}_{\text {critical }}$ (present) \\
\hline 100 & 0 & 9.8696 & 9.8698 \\
& 0.5 & 9.4055 & 9.4056 \\
& 1 & 8.9830 & 8.9832 \\
20 & 2 & 8.2426 & 8.2427 \\
& 0 & 9.8696 & 9.8698 \\
& 0.5 & 9.4055 & 9.4056 \\
& 1 & 8.9830 & 8.9832 \\
& 2 & 8.2426 & 8.2427
\end{tabular}




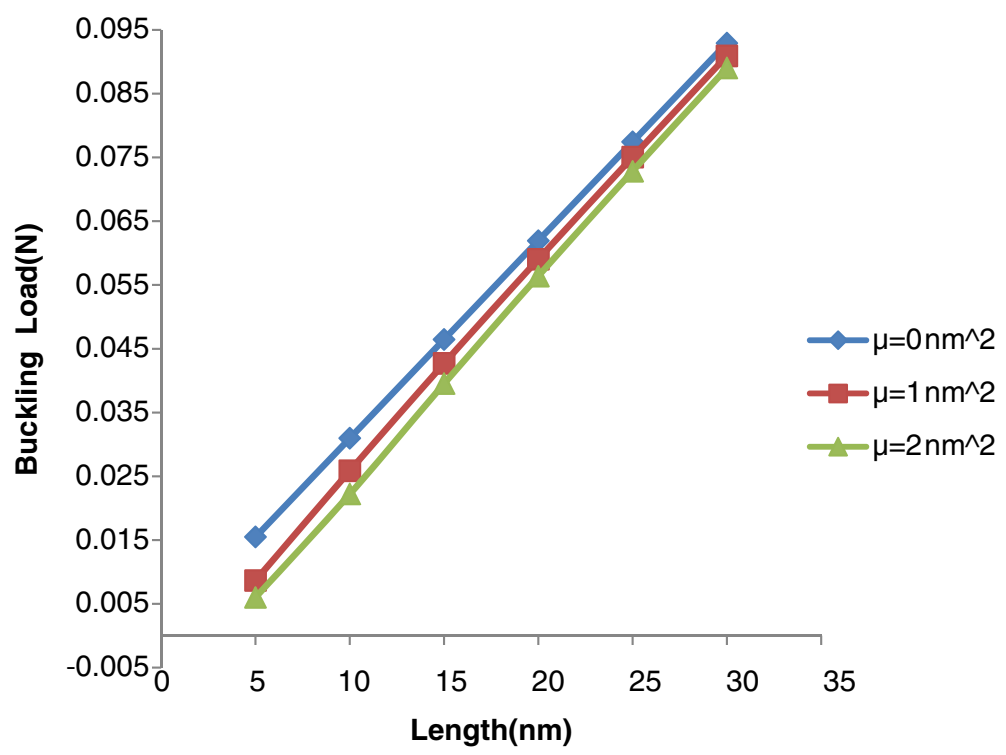

Figure 2. Variation of buckling load with the length of a square graphene sheet for the SSSS boundary condition for various values of the non-local parameter.

\section{Results and discussions}

\subsection{Validation}

The governing equation for buckling of non-local graphene sheets is written in Eq. (10). It can be seen that by putting $\mu=0$ in this equation, traditional local elastic plate equations are obtained.

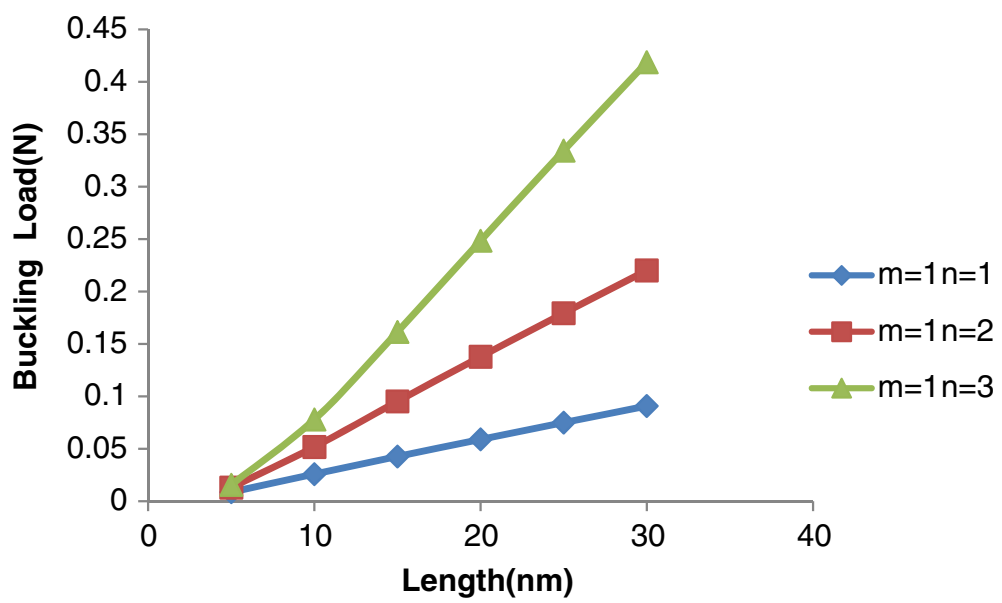

Figure 3. Variation of buckling load with length of a square graphene sheet for the SSSS boundary condition for various modes of buckling. 


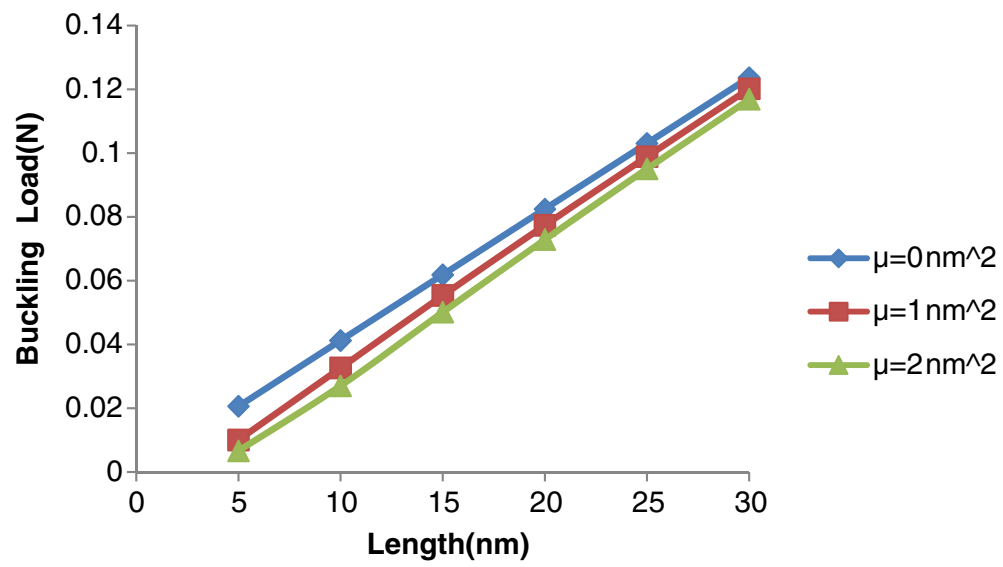

Figure 4. Variation of buckling load with the length of a square graphene sheet for the SSSC boundary condition for various values of the non-local parameter.

Further, using $\frac{E h^{3}}{12\left(1-v^{2}\right)}=E I$ and $b=\infty$, and all four sides simply supported in the current analysis, non-local solutions for buckling of simply supported beam are obtained. A beam with the following material properties and geometrical dimensions are considered: Young's modulus $E=30 \mathrm{GPa}$, length $L=10 \mathrm{~m}$, height h is varied, Poisson's ratio $v=0.3$. Critical buckling loads obtained are expressed in non-dimensional form as $\bar{P}_{\text {critical }}=P_{\text {critical }} \times \frac{L^{2}}{E I}$ (Reddy 2007). Non-dimensional critical buckling loads are calculated using the current analysis for the above mentioned beam. The results are compared with the results of Reddy (2007) for the beam in table 1. In this table, one could observe that the present critical buckling loads results agree with those reported in Reddy (2007).

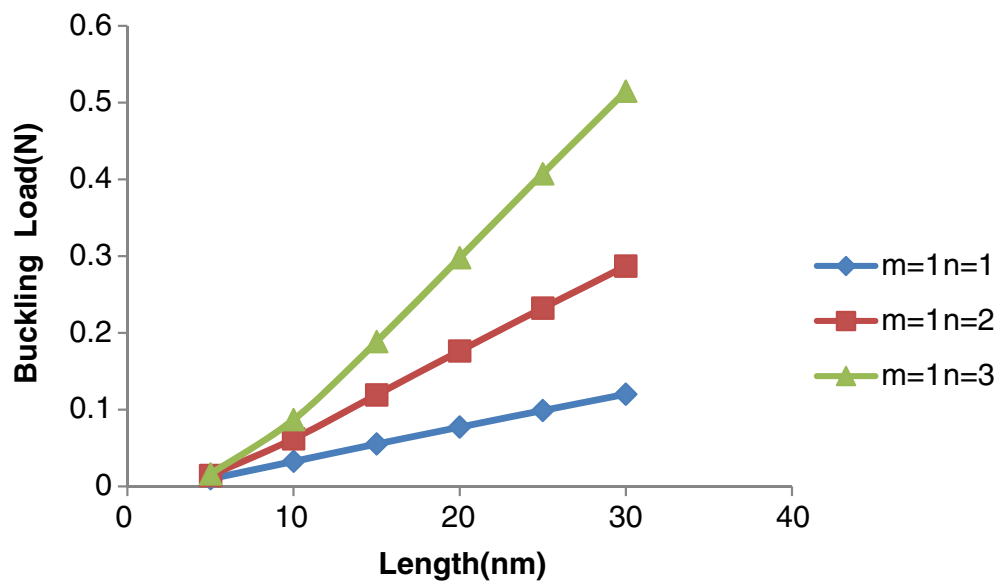

Figure 5. Variation of buckling load with length of a square graphene sheet for the SSSC boundary condition for various modes of buckling. 


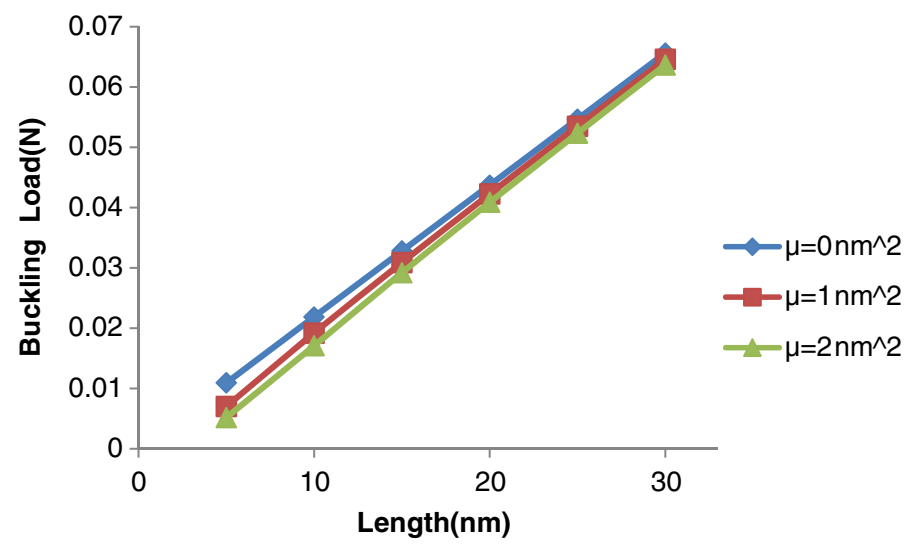

Figure 6. Variation of buckling load with the length of a square graphene sheet for the SSSF boundary condition for various values of the non-local parameter.

For the purpose of analysis of graphene sheets with various boundary conditions, a square graphene sheet having $\mathrm{E}=1.765 \mathrm{TPa}$ and $v=0.3$ are considered.

Buckling load ratio is defined as

$$
\text { Buckling load ratio }=\frac{\text { Critical buckling load calculated using non-local theory }}{\text { Critical buckling load calculated using local theory }} \text {. }
$$

\subsection{SSSS Boundary condition}

Variation of buckling load with the length of the square graphene sheet for the SSSS boundary condition with various values of the non-local parameter is depicted in figure 2. Effect of higher modes of buckling for the SSSS boundary condition is plotted in figure 3 .

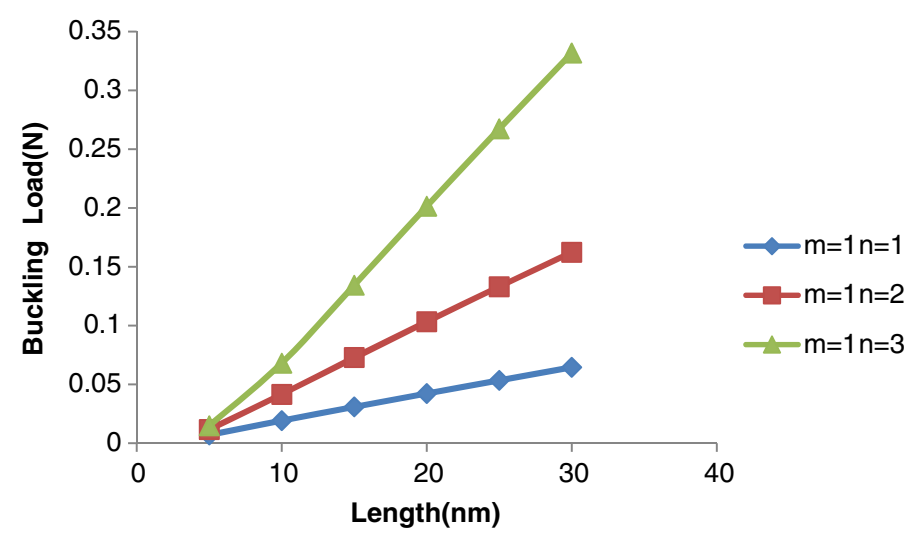

Figure 7. Variation of buckling load with length of a square graphene sheet for the SSSF boundary condition for various modes of buckling. 


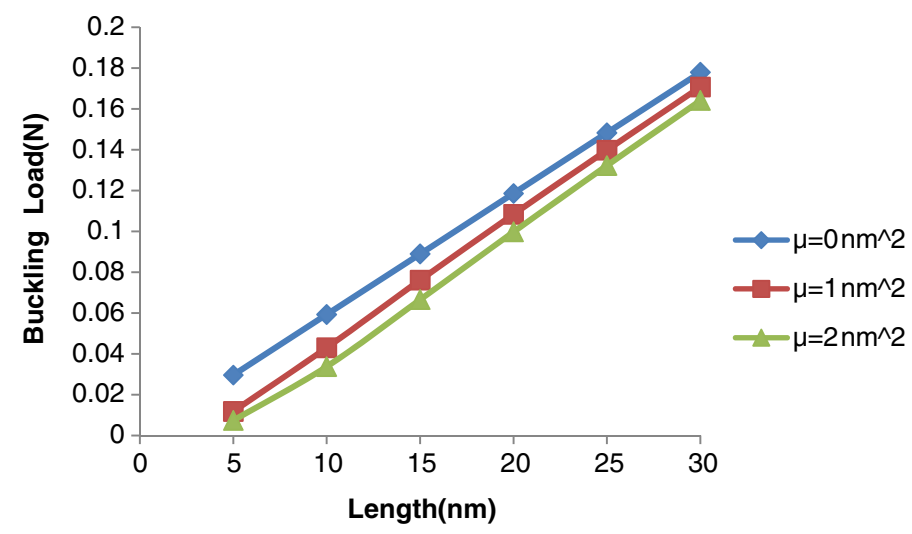

Figure 8. Variation of buckling load with the length of a square graphene sheet for the SCSC boundary condition for various values of the non-local parameter.

\subsection{SSSC Boundary condition}

Variation of buckling load with the length of the square graphene sheet for the SSSC boundary condition with various values of the non-local parameter is depicted in figure 4. Effect of higher modes of buckling for the SSSC boundary condition is plotted in figure 5.

\subsection{SSSF Boundary condition}

Variation of buckling load with the length of the square graphene sheet for the SSSF boundary condition with various values of the non-local parameter is depicted in figure 6. Effect of higher modes of buckling for the SSSF boundary condition is plotted in figure 7 .

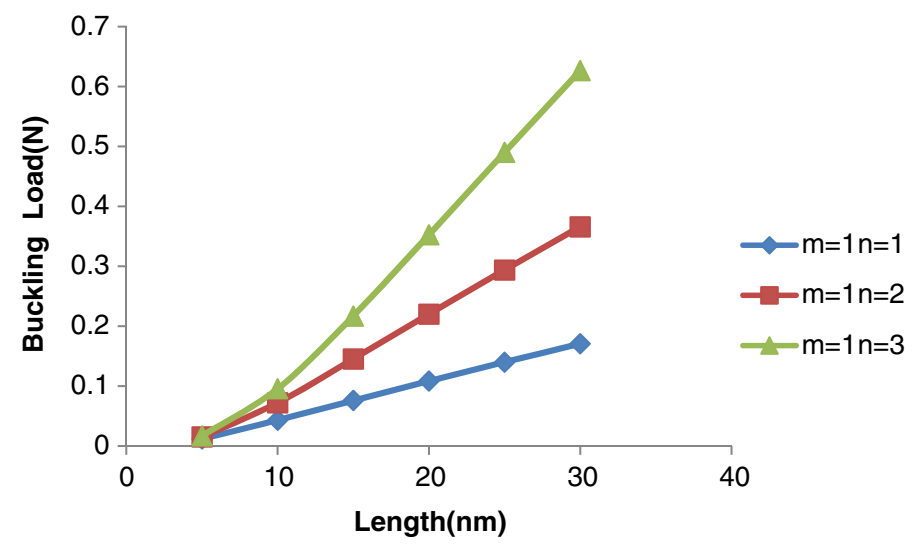

Figure 9. Variation of buckling load with length of a square graphene sheet for the SCSC boundary condition for various modes of buckling. 


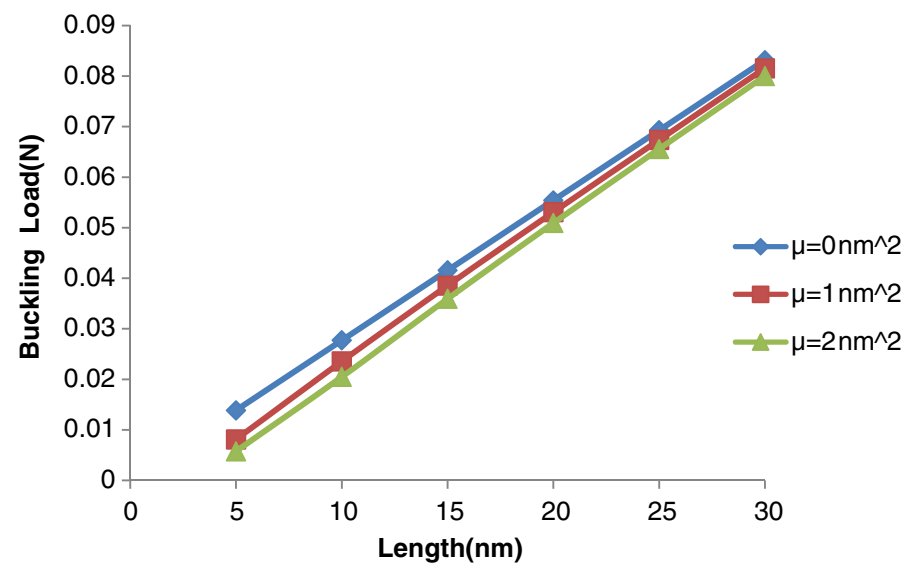

Figure 10. Variation of buckling load with the length of a square graphene sheet for the SCSF boundary condition for various values of the non-local parameter.

\subsection{SCSC Boundary condition}

Variation of buckling load with the length of the square graphene sheet for the SCSC boundary condition with various values of the non-local parameter is depicted in figure 8. Effect of higher modes of buckling for the SCSC boundary condition is plotted in figure 9 .

\subsection{SCSF boundary condition}

Variation of buckling load with the length of the square graphene sheet for the SCSF boundary condition with various values of the non-local parameter is depicted in figure 10. Effect of higher modes of buckling for the SCSF boundary condition is plotted in figure 11.

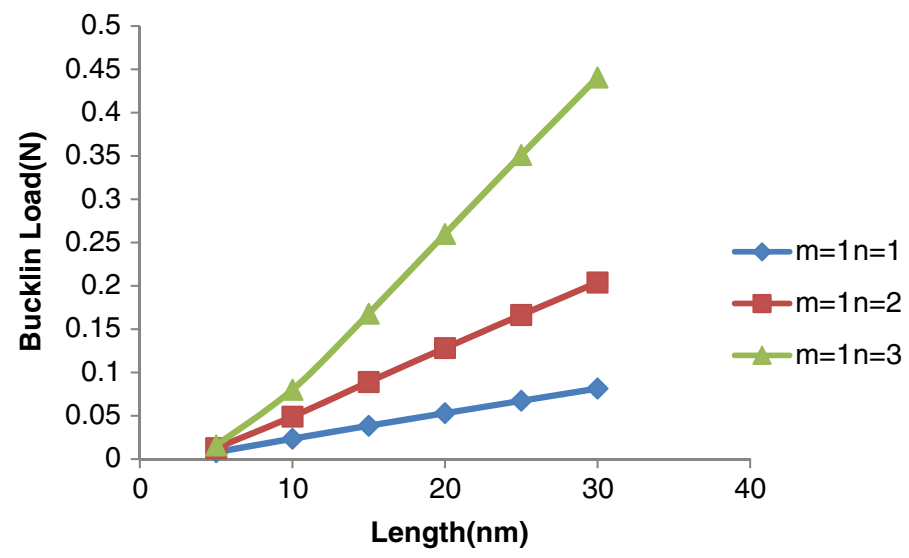

Figure 11. Variation of buckling load with length of a square graphene sheet for the SCSF boundary condition for various modes of buckling. 


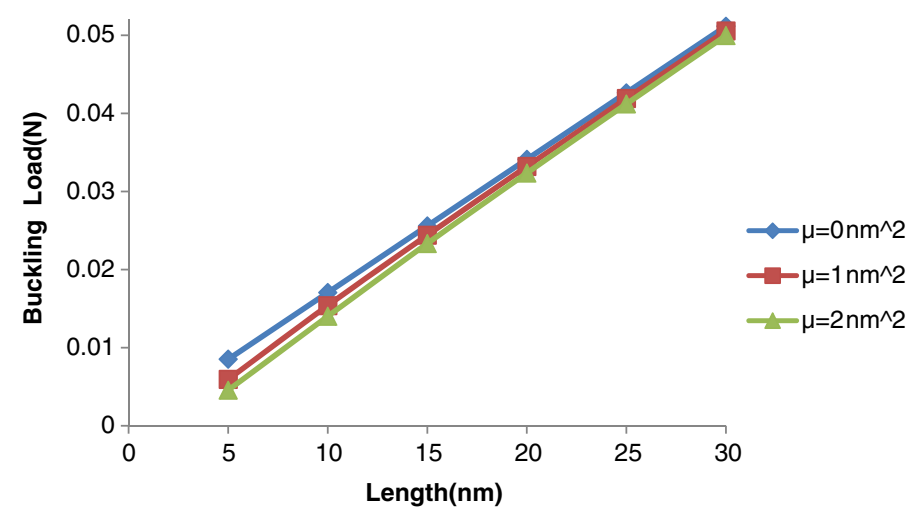

Figure 12. Variation of buckling load with the length of a square graphene sheet for the SFSF boundary condition for various values of the non-local parameter.

\subsection{SFSF Boundary condition}

Variation of buckling load with the length of the square graphene sheet for the SFSF boundary condition with various values of the non-local parameter is depicted in figure 12. Effect of higher modes of buckling for the SFSF boundary condition is plotted in figure 13.

For the graphene sheet with the above six boundary condition, it is observed that the buckling load decreases with decrease in the graphene sheet length, and approaches the local theory value as the length is increased. Further, it can also be seen that the buckling load is smaller for a larger value of the non-local parameter. Besides, it can also be inferred that the buckling load is smaller for higher modes of buckling. Hence the non-local effect has more prominence for higher buckling modes.

Variation of buckling load with length of the graphene sheet for $\mu=2 \mathrm{~nm}^{2}$ and various boundary conditions is shown in figure 14. From this figure, it can be observed that the buckling load is largest for the graphene sheet with SFSF boundary condition and the smallest for

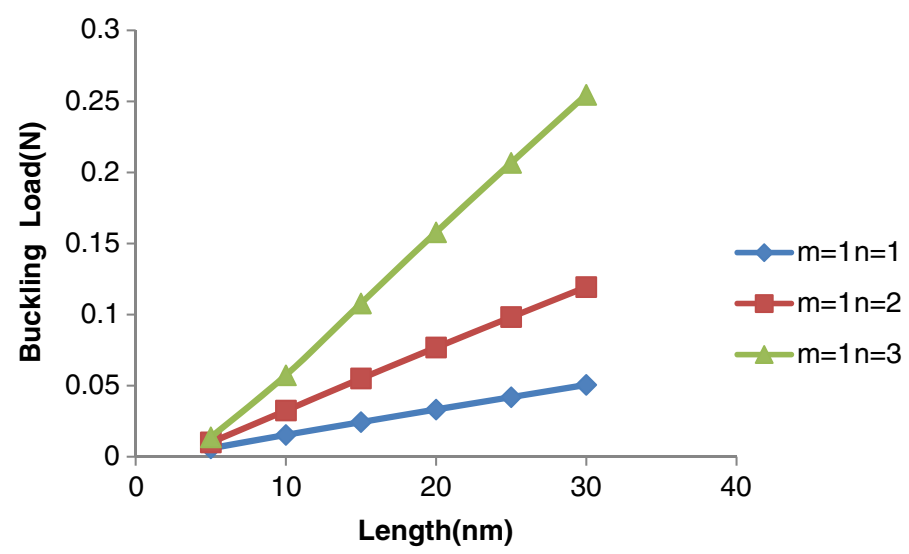

Figure 13. Variation of buckling load with length of a square graphene sheet for the SFSF boundary condition for various modes of buckling. 


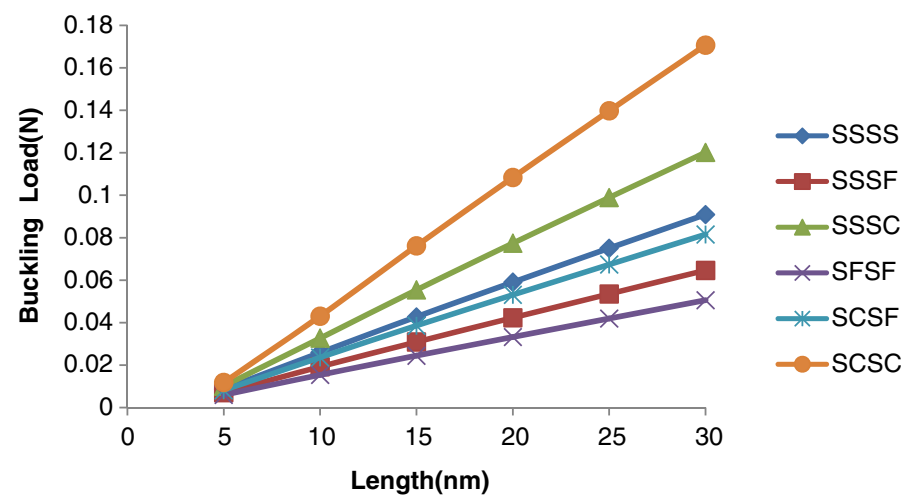

Figure 14. Variation of buckling load with length of a square graphene sheet for $\mu=2 \mathrm{~nm}^{2}$ and various boundary conditions.

the graphene sheet with SCSC boundary condition. The buckling load for graphene sheets with various boundary conditions are in the following order.

$$
\mathrm{SFSF}>\mathrm{SSSF}>\mathrm{SCSF}>\mathrm{SSSS}>\mathrm{SSSC}>\mathrm{SCSC} .
$$

\section{Conclusions}

In the present work, Levy's solution is used for solving the derived non-local elasticity governing equation for a graphene sheet. Levy's solution is carried out for six boundary conditions and the results are reported. The obtained buckling loads have been validated for the SSSS boundary condition and are found to be in agreement. It has been found that the non-local effect becomes more prominent as the graphene sheet size decreases. Further, the buckling load decreases with increase in the non-local parameter for all the six boundary conditions considered. Also, the buckling load ratio is smaller for higher modes of buckling. The buckling load ratios have been observed in the following order for the six boundary conditions on the graphene sheet.

$$
\mathrm{SFSF}>\mathrm{SSSF}>\mathrm{SCSF}>\mathrm{SSSS}>\mathrm{SSSC}>\mathrm{SCSC} .
$$

There is a significant change in the buckling results associated with small size graphene sheet, higher modes and larger non-local parameters. Thus, one should include non-local elasticity theory for the buckling analysis of the grapheme sheets.

\section{Notations}

$a, b$

$D$

E

G

$h$

$L$
Length and breadth of the graphene sheet, respectively

Bending rigidity of the isotropic graphene sheet

Young's modulus of the graphene sheet material

Shear modulus of the graphene sheet material

Thickness of the graphene sheet

Length of a square graphene sheet 


$M_{1}^{x x}, M_{1}^{y y}, M_{1}^{x y}$
$N_{0}^{x x}, N_{0}^{y y}, N_{0}^{x y}$
$q$
$S(x)$
$\boldsymbol{t}$
$u, v$
$w$
$\varepsilon_{x x}, \varepsilon_{y y}, \varepsilon_{z z}, \varepsilon_{x y}, \varepsilon_{y z}, \varepsilon_{x z}$
$\mu$
$v$
$\sigma_{x x}, \sigma_{y y}, \sigma_{z z}, \sigma_{x y}, \sigma_{y z}, \sigma_{x z}$
$P_{c r i t i c a l}$
$\nabla^{2}$

Moment resultants

In-plane force resultants

Transverse distributed load

Fourth order elasticity tensor

Macroscopic stress tensor

Displacement of the point $(x, y, 0)$ of graphene sheet along $x$ and $y$ axis, respectively.

Deflection of the graphene sheet at point $(x, y)$

Strain components

Non-local parameter

Poisson's ratio of the graphene sheet material

Stress components

Buckling loads of single layered graphene sheet

Laplacian operator in two-dimensional Cartesian coordinate system.

\section{Acknowledgements}

Author thanks Mr. M Pant, undergraduate student of Indian Institute of Technology Kharagpur for some computational results.

\section{References}

Ball P 2001 Roll up for the revolution. Nature 414: 142-144

Baughman R H, Zakhidov A A and de Heer W A 2002 Carbon nanotubes-the route toward applications. Science 297(5582): 787-792

Behfar K and Naghdabadi R 2005 Nanoscale vibrational analysis of a multi-layered graphene sheet embedded in an elastic medium. Compos. Sci. Tech. 65(7-8): 1159-1164

Duan W H and Wang C M 2007 Exact solutions for axisymmetric bending of micro/nanoscale circular plates based on non-local plate theory. Nanotechnology 18(38): 385704

Eringen A C 1983 On differential equations of nonlocal elasticity and solutions of screw dislocation and surface waves. J. Appl. Phys. 54: 4703

Eringen A C 2002 Nonlocal continuum field theories. New York: Springer Verlag

Ghorbanpour Arani A, Rahmani R and Arefmanesh A 2008 Elastic buckling analysis of single-walled carbon nanotube under combined loading by using the ANSYS software. Physica E 40(7): 2390-2395

Ghorbanpour Arani A, Mohammadimehr M, Arefmanesh A and Ghasemi A 2010 Transverse vibration of short carbon nanotubes using cylindrical shell and beam models. J. Mech. Eng. Sci. 224(3): 745-756

Ghorbanpour Arani A, Mohammadimehr M, Saidi A R, Shogaei S and Arefmanesh A 2011 Thermal buckling analysis of double walled carbon nano tubes considering the small scale effect. J. Mech. Eng. Sci. 225(1), 248-256

Gibson R F, Ayorinde E O and Wen Y-F 2007 Vibrations of carbon nanotubes and their composites: A review. Compos. Sci. Technol. 67(1): 1-28

Hsu M 2010 Dynamic analysis of multi-walled carbon nanotubes using the differential quadrature method. J. Mech. Eng. Sci. 224(3): 731-744

Iijima S 1991 Helical microtubules of graphitic carbon. Nature 354: 56-58

Karaoglu P and Aydogdu M 2010 On the forced vibration of carbon nanotubes via a non-local EulerBernoulli beam model. J. Mech. Eng. Sci. 224(2): 497-503

Kong X Y, Ding Y, Yang R and Wang Z L 2004 Single-crystal nanorings formed by epitaxial self-coiling of polar nanobelts. Science 303(5662): 1348-1351 
Kroto H W 1985 C60: Buckminsterfullerene. Nature 318: 162-163

Kitipornchai S, He X Q and Liew K M 2005 Continuum model for the vibration of multilayered graphene sheets. Phys. Rev. B 72: 075443

Liew K M, He X Q and Kitipornchai S 2006 Predicting nanovibration of multi-layered graphene sheets embedded in an elastic matrix. Acta Mater. 54(16): 4229-4236

Lu P, Lee H P, Lu C and Zhang P Q 2007 Application of nonlocal beam models for carbon nanotubes. Int. J. Solids Struct. 44(16): 5289-5300

Murmu T and Pradhan S C 2008 Buckling analysis of beam on Winkler foundation by using MDQM and nonlocal theory. J. Aero. Sci. Technol. 60(3): 206-215

Novoselov K S, Geim A K, Morozov S V, Jiang D, Zhang Y, Dubonos S V, Grigorieva I V and Firsov A A 2004 Electric field effect in atomically thin carbon films. Science 306(5696): 666-669

Peddieson J, Buchanan G R and McNitt R P 2003 Application of nonlocal continuum models to nanotechnology. Int. J. Eng. Sci. 41(3-5): 305-312

Phadikar J K and Pradhan S C 2008 Nonlinear finite element model of single wall carbon nano tubes. J. Inst. Eng. (India) Metallurgy Mater. Eng. Div. 89: 3-8

Phadikar J K and Pradhan S C 2009 Bending, vibration and buckling analysis of non homogenous nanotubes using nonlocal elasticity theory and GDQ method. Struct. Eng. Mech. 33(2): 193-213

Pradhan S C 2009 Buckling of single layer graphene sheet based on nonlocal elasticity and higher order shear deformation theory. Phys. Lett. A 373: 4182-4188

Pradhan S C 2012 Nonlocal finite element analysis and small scale effects of CNTs with Timoshenko beam theory. Finite Elements in Analysis and Design 50: 8-20

Pradhan S C and Murmu T 2009a Small scale effect on the buckling of single-layered graphene sheets under bi-axial compression via nonlocal continuum mechanics. Comput. Mat. Sci. 47: 268-274

Pradhan S C and Murmu T 2009b Differential quadrature method for vibration analysis of beam on Winkler foundation based on nonlocal elastic theory. J. Inst. Eng. (India) Metallurgy Mater. Eng. Div. 89: $3-12$

Pradhan S C and Murmu T 2009c Small-scale effect on the free in-plane vibration of nanoplates by nonlocal continuum model. Physica E 41(8): 1628-1633

Pradhan S C and Murmu T 2009d Thermo-mechanical vibration of FGM sandwich beam under variable elastic foundation using differential quadrature method. J. Sound Vib. 321: 342-362

Pradhan S C and Murmu T 2010a Application of nonlocal elasticity and DQM in the flapwise bending vibration of a rotating nanocantilever. Physica E 42: 1944-1949

Pradhan S C and Murmu T 2010b Small scale effect on the buckling analysis of single-layered graphene sheet embedded in an elastic medium based on nonlocal plate theory. Physica E 42(5): 1293-1301

Pradhan S C and Phadikar J K 2009a Bending, buckling and vibration analyses of nonhomogeneous nanotubes using GDQ and nonlocal elasticity theory. Struct. Eng. Mech. Int. J. 33(2): 193-213

Pradhan S C and Phadikar J K 2009b Small scale effect on vibration of embedded multilayered graphene sheets based on nonlocal continuum models. Phys. Lett. A 373(11): 1062-1069

Pradhan S C and Phadikar J K 2010 Scale effect and buckling analysis of multilayered graphene sheets based on nonlocal continuum mechanics. J. Comput. Theor. Nanosci. 7: 1-7

Pradhan S C and Sahu B 2010 Vibration of single layer graphene sheet based on nonlocal elasticity and higher order shear deformation theory. J. Comput. Theor. Nanosci. 7(6): 1042-1050

Pradhan S C and Sarkar A 2009 Analyses of tapered FGM beams with nonlocal theory. Struct. Eng. Mech. Int. J. 32(6): 811-833

Reddy J N 1997 Mechanics of laminated composite plates: Theory and analysis. Boca Raton, FL: Chemical Rubber Company

Reddy J N 2007 Nonlocal theories for bending, buckling and vibration of beams. Int. J. Eng. Sci. 45(2-8): 288-307

Stankovich S, Dikin D A, Dommett G H B, Kohlhaas K M, Zimney E J, Stach E A, Piner R D, Nguyen S T and Ruoff R S, 2006 Graphene based composite materials. Nature 442: 282-286

Thostenson E T, Ren Z and Chou T-W 2001 Advances in the science and technology of carbon nanotubes and their composites: a review. Compos. Sci. Tech. 61(13): 1899-1912 
Timoshenko S P and Gere J M 1985 Theory of elastic stability, Singapore: McGraw Hill International Book Company

Wang L, Ni Q, Li M and Qian Q 2008 The thermal effect on vibration and instability of carbon nanotubes conveying fluid. Physica E 40(10): 3179-3182

Wang Q and Vardan V K 2006 Wave characteristics of carbon nanotubes. Int. J. Solids Struct. 43(2): 254265 